\title{
Australian Government
}

Department of Health

$2022 \cdot$ Volume 46

\section{Communicable Diseases Intelligence \\ Nocardiosis in the Illawarra-Shoalhaven \\ Regions from 2010 to 2019}

Darcy Gray, Simeon Crawford, Peter Newton

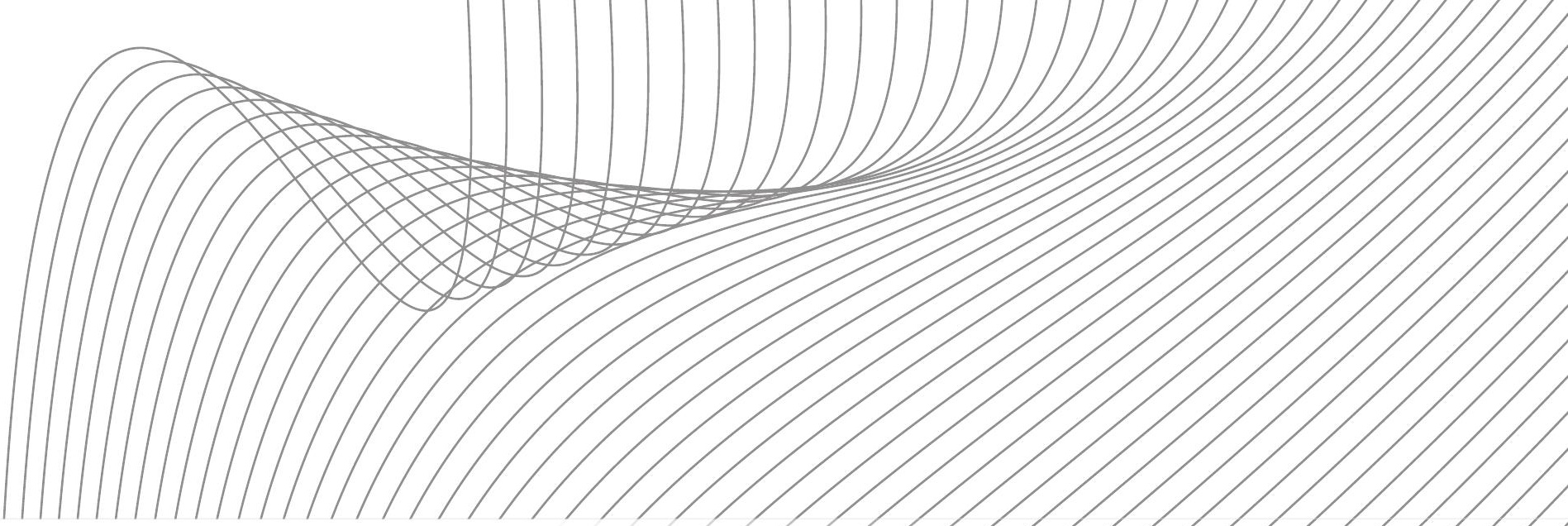

https://doi.org/10.33321/cdi.2022.46.7

Electronic publication date: 16/02/2022 


\section{Communicable Diseases Intelligence}

ISSN: 2209-6051 Online

This journal is indexed by Index Medicus and Medline.

Creative Commons Licence - Attribution-NonCommercial-

NoDerivatives CC BY-NC-ND

(C) 2022 Commonwealth of Australia as represented by the Department of Health

This publication is licensed under a Creative Commons Attribution - Non-Commercial NoDerivatives 4.0 International Licence from https://creativecommons.org/licenses/by-nc-nd/4.0/legalcode (Licence). You must read and understand the Licence before using any material from this publication.

\section{Restrictions}

The Licence does not cover, and there is no permission given for, use of any of the following material found in this publication (if any):

- the Commonwealth Coat of Arms (by way of information, the terms under which the Coat of Arms may be used can be found at www.itsanhonour.gov.au);

- any logos (including the Department of Health's logo) and trademarks;

- any photographs and images;

- any signatures; and

- any material belonging to third parties.

\section{Disclaimer}

Opinions expressed in Communicable Diseases Intelligence are those of the authors and not necessarily those of the Australian Government Department of Health or the Communicable Diseases Network Australia. Data may be subject to revision.

\section{Enquiries}

Enquiries regarding any other use of this publication should be addressed to the Communication Branch, Department of Health, GPO Box 9848, Canberra ACT 2601, or via e-mail to: copyright@health.gov.au

\section{Communicable Diseases Network Australia}

Communicable Diseases Intelligence contributes to the work of the Communicable Diseases Network Australia.

http://www.health.gov.au/cdna
Communicable Diseases Intelligence

(CDI) is a peer-reviewed scientific journal published by the Office of Health Protection and Response, Department of Health. The journal aims to disseminate information on the epidemiology, surveillance, prevention and control of communicable diseases of relevance to Australia.

\section{Editor}

Jennie Hood

\section{Deputy Editor}

Simon Petrie

\section{Design and Production} Kasra Yousefi

\section{Editorial Advisory Board}

David Durrheim, Mark Ferson, John Kaldor, Martyn Kirk and Linda Selvey

\section{Website}

http://www.health.gov.au/cdi

\section{Contacts}

$\mathrm{CDI}$ is produced by the Office of Health Protection and Response, Australian Government Department of Health, GPO Box 9848, (MDP 6) CANBERRA ACT 2601

\section{Email:}

cdi.editor@health.gov.au

\section{Submit an Article}

You are invited to submit your next communicable disease related article to the Communicable Diseases Intelligence (CDI) for consideration. More information regarding $\mathrm{CDI}$ can be found at:

http://health.gov.au/cdi.

Further enquiries should be directed to: cdi.editor@health.gov.au. 


\title{
Original article
}

\section{Nocardiosis in the Illawarra-Shoalhaven region from 2010 to 2019}

\author{
Darcy Gray, Simeon Crawford, Peter Newton
}

\begin{abstract}
Nocardia species are a diverse group of gram-positive bacteria that are encountered as pathogens in clinical practice. They are ubiquitous environmental organisms classically causing opportunistic infections in immunocompromised individuals. This case series describes the epidemiological, microbiological and clinical features of 26 culture-proven cases of nocardiosis presenting to the public healthcare service in a regional area of New South Wales over a ten year period. These cases involved ten distinct species of Nocardia and appeared to demonstrate increasing incidence over time.
\end{abstract}

The majority of Nocardia isolates (88\%) were grown on conventional bacteriological media and speciation was primarily via $16 \mathrm{~S}$ rRNA sequencing and/or matrix assisted laser desorption ionisationtime of flight mass spectrometry. Similar to other Australian Nocardia studies, the isolates seen in this case series were universally susceptible to trimethoprim-sulfamethoxazole. This was reflected in treatment data, with all but one patient receiving this agent as part of their antibiotic regimen.

While similar case series have been reported from other Australian states, there are no published clinical studies of nocardiosis in New South Wales. Given the nature of Nocardia species as an environmental saprophyte, this study examined the association between incidence of nocardiosis and regional geographic or climatic variation but was unable to demonstrate a statistically significant correlation.

Keywords: Nocardia; nocardial infection; Australia; microbiology

\section{Introduction}

Nocardia species are a diverse group of filamentous gram-positive aerobic bacteria of the order Actinomycetales. They are most commonly associated with opportunistic infections, often disseminated, in immunocompromised patients. They can also cause localised infection following direct inoculation, usually without dissemination, in immunocompetent

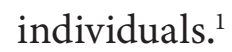

These bacteria are ubiquitous within the environment and are present in soil, water and organic material. ${ }^{2}$ They have a variable geographic distribution. Data on infections in Australia is limited and comprised of case series and small observational studies. While previous reports have defined the pattern of nocardiosis in the Northern Territory, ${ }^{3}$ Queensland, ${ }^{4}$ Victoria, ${ }^{5,6}$ and the Australian Capital Territory, ${ }^{7}$ none have examined the presentations related to Nocardia infections in New South Wales (NSW).

The Illawarra-Shoalhaven region extends from Helensburgh on the southern outskirts of Sydney to North Durras on the NSW South Coast. This region had a population of approximately 400,000 in $2015 ;{ }^{8,9}$ it comprises a diverse range of human environments from major metropolitan to rural areas. The Illawarra Shoalhaven Local Health District (ISLHD) provides public healthcare services to this region. 
Wollongong Hospital is the tertiary referral hospital and location of the NSW Health Pathology microbiology laboratory serving ISLHD. In this study we sought to examine all cases of nocardiosis presenting to ISLHD services over the ten-year period from 2010 to 2019, to understand the local epidemiology, microbiology and clinical characteristics of Nocardia infections compared to that described in other studies. We also assessed the association between incidence of nocardiosis in ISLHD and regional rainfall patterns.

\section{Methods}

Cases of culture-confirmed nocardiosis were identified through interrogation of the NSW Health Pathology Wollongong Hospital microbiology laboratory records for the period 20102019. The NSW Health Pathology reference laboratories, Institute for Clinical Pathology and Medical Research (Westmead Hospital) and Australian National Reference Laboratory in Medical Mycology (Royal North Shore Hospital), were also canvassed for isolates sent to these sites from ISLHD during this time period. Additional cases were identified through a search of the clinical records of the Department of Infectious Diseases at Wollongong Hospital and Shoalhaven District Memorial Hospital.

Routine cultures of swabs and respiratory samples were inoculated, as a minimum, onto horse blood agar and MacConkey agar and incubated for two days. Tissue samples, normally sterile body fluids and positive blood culture subcultures were additionally inoculated onto chocolate agar and incubated routinely for three days. If Nocardia culture was requested or branching gram-positive bacilli were seen on direct specimen microscopy then direct, modified acid-fast (Ziehl-Neelsen) stain and standard acid-fast (Ziehl-Neelsen) stains were performed/ examined and blood agar plate cultures were incubated aerobically for seven days. Blood cultures were inoculated into BD BACTEC Plus Aerobic/F (Becton Dickinson) and processed with the BD BACTEC 9240 (Becton Dickinson) system with a routine incubation period of five days. If mycobacterial culture was requested the samples were processed by the NSW Health Pathology Mycobacteriology laboratory at St George Hospital, Kogarah, NSW Australia. Samples were inoculated onto two LowensteinJensen agar slopes (with and without pyruvate) and a Mycobacteria Growth Indicator Tube (Becton Dickinson) and incubated routinely for eight weeks. No changes in the laboratory methods for Nocardia species culture were made during the study period.

Culture isolates were presumptively identified as Nocardia species based on strict aerobic growth, colony morphology, Gram stain morphology and positive modified acid-fast (ZiehlNeelsen) stain and negative standard acid-fast (Ziehl-Neelsen) stain. All presumptive Nocardia isolates were forwarded to either of the two above-mentioned NSW Health Pathology reference laboratories for confirmation, species identification, and antimicrobial susceptibility testing. Species identification was made using $16 \mathrm{~S}$ rRNA sequencing and/or matrixassisted laser desorption ionisation-time of flight mass spectrometry (MALDI-TOF MS). Antimicrobial susceptibilities were determined using a commercially-available broth microdilution method, Sensititre ${ }^{\mathrm{TM}}$ RAPMYCO (Trek Diagnostic Systems, Biocentric), with the exception of meropenem susceptibility which was determined using Epsilometer test (E-Test ${ }^{\circledR}$, bioMérieux). Where possible, susceptibilities were interpreted using Clinical and Laboratory Standards Institute (CLSI) breakpoints.

For all patients with cultures positive for Nocardia species, a search of the ISLHD electronic medical record was conducted to compile relevant data including patient demographics, risk factors for nocardiosis, clinical features of infection, treatment and outcome data.

Limited disease was defined as: either (i) skin and soft tissue infection with or without contiguous osteomyelitis, or (ii) pulmonary disease, both without evidence of other organ involvement. Disseminated disease was defined as disease involving two or more organ systems, 
bacteraemia, or isolated central nervous system (CNS) disease. ${ }^{5}$ Survival calculations were based on documented evidence that a patient was alive or deceased. If no evidence was available regarding a patient after a given time point, they were excluded from analysis at that time point. Local incidence was calculated using the number of nocardiosis cases over the ten-year study period and postcode population data from the 2016 Australian Census, ${ }^{10}$ expressed as cases per 100,000 persons. Case numbers were compared to Bureau of Meteorology total annual rainfall data for Jamberoo ${ }^{11}$ (a geographically-central area of the region). Statistical correlation between rainfall and case numbers was compared using a Pearson product-moment linear regression coefficient. Statistical testing was then performed based on transforming this coefficient into a Student's T statistic, based on a presumption that both variables were from an uncorrelated bivariate normal distribution, which was itself tested using a Shapiro-Wilk test for each variable.

The data were deidentified and aggregated with analysis of descriptive statistics performed using the Microsoft Excel platform. The study underwent ethics approval through the joint University of Wollongong/Illawarra Shoalhaven Local Health District Human Ethics Review Committee (approval number: ETH10680).

\section{Results}

\section{Epidemiology and clinical features}

Twenty-six cases of culture-proven Nocardia sp. infection were identified. Patient demographic data and predisposing conditions are displayed in Table 1. Since 2010, cases of nocardiosis presenting to ISLHD facilities involved ten distinct species (Table 2), resulting in a variety of clinical presentations including skin and soft tissue infection with or without osteomyelitis $(\mathrm{N}=$ 8), pulmonary disease $(\mathrm{N}=8)$ or disseminated disease $(\mathrm{N}=9)$ (Figure 1). One case had limited clinical data available and was excluded from the treatment and outcomes analyses. Two cases had incomplete antimicrobial susceptibility data (with one of these having no available antimicrobial susceptibilities). No patients included in the study were receiving antibiotic prophylaxis with activity against Nocardia species (e.g. trimethoprim-sulfamethoxazole) prior to presentation. Median and range displayed for prednisolone dose was calculated from data available in the medical record; doses were unavailable for three patients.

While pulmonary and disseminated disease were caused by several different Nocardia species (Figure 2), locally-acquired skin and soft tissue infection was caused almost exclusively by $N$. brasiliensis. One patient experienced skin and soft tissue infection with contiguous osteomyelitis due to N. rhamnosiphila acquired by traumatic inoculation sustained overseas; another case of soft tissue infection caused by $N$. thailandica was again related to direct inoculation.

\section{Pulmonary imaging findings}

Twelve patients had pulmonary involvement (either isolated or in the setting of disseminated disease). Chest computerised tomography (CT) reports were available for analysis of the imaging findings in 11 patients. Chest CT in 2/11 (18\%) patients demonstrated single nodules and in $5 / 11(45 \%)$ demonstrated multiple nodules; in $3 / 7$ patients with pulmonary nodules there was cavitation. Consolidation was present on imaging in $3 / 11(27 \%)$ patients, of whom two had an associated pleural effusion. The remaining patient had mediastinal lymphadenopathy without lung nodules or consolidation.

\section{Local epidemiology}

Local incidence data to postcode level (Figure 3) demonstrates that the highest rates of nocardiosis (approximately 19 cases per 100,000 persons over the 10 year study period) occurred in the rural southern Shoalhaven region as well as in the postcodes around Lake Illawarra (just south of Wollongong). 
Table 1: Demographic information and epidemiological risk factors

\begin{tabular}{|c|c|c|}
\hline Characteristic & Number of patients & $\%$ \\
\hline Female & $10 / 26$ & 39 \\
\hline Median age in years [Range] & 67 [11-92] & \\
\hline \multicolumn{3}{|l|}{ Risk factors } \\
\hline Haematological malignancy & $5 / 26$ & 19 \\
\hline $\begin{array}{l}\text { Corticosteroid therapy: } \\
\text { Median dose (mg/day prednisolone) } \\
\text { [Range] }\end{array}$ & $\begin{array}{c}11 / 26 \\
12.5 \\
{[7.5-25]}\end{array}$ & 42 \\
\hline Other immunosuppression & $6 / 26$ & 23 \\
\hline Chronic lung disease & $5 / 26$ & 19 \\
\hline Type 2 diabetes mellitus & $5 / 26$ & 19 \\
\hline End stage renal disease on haemodialysis & $1 / 26$ & 4 \\
\hline Renal transplant & $3 / 26$ & 12 \\
\hline HIV & $1 / 26$ & 4 \\
\hline Trauma/inoculation & $3 / 26$ & 11 \\
\hline No identified risk factor & $3 / 26$ & 11 \\
\hline
\end{tabular}

Figure 1: Incident cases of culture-proven nocardiosis presenting to the Illawarra Shoalhaven Local Health District

8

目 Lymph node only 国 Pulmonary Disseminated $\quad$ Skin and soft tissue

7

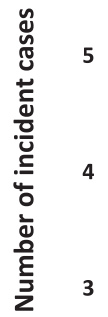

2

20092010
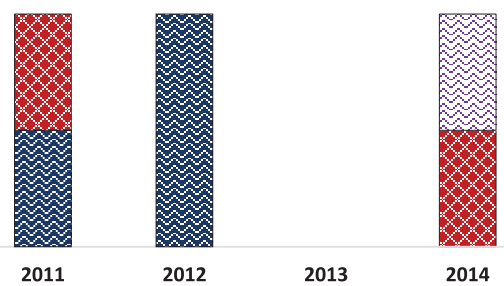

2013

2014

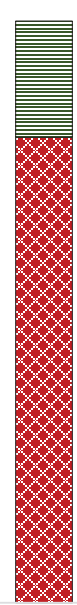

Year 
Table 2: Incident cases of culture proven nocardiosis presenting to the Illawarra Shoalhaven Local Health District classified by Nocardia species during the ten-year study period

\begin{tabular}{l|c|}
\hline Species & $\begin{array}{c}\text { Number of } \\
\text { isolates }\end{array}$ \\
\hline Nocardia brasiliensis & 6 \\
Nocardia farcinica & 6 \\
Nocardia cyriacigeorgica & 5 \\
Nocardia nova & 3 \\
Nocardia paucivorans & 1 \\
\hline Nocardia vinacea & 1 \\
\hline Nocardia sp. (N. gamkensis or N. exalbida) & 1 \\
\hline Nocardia beijingenesis & 1 \\
\hline Nocardia rhamnosiphila & 1 \\
\hline Nocardia thailandica & 1 \\
\hline
\end{tabular}

Annual nocardiosis case numbers in the Illawarra-Shoalhaven region were compared to average annual rainfall in Jamberoo (Figure 4) to examine for any possible linkage between incidence of infection and climatic factors. This had a correlation coefficient of $-0.34(p=0.2)$ and thus did not demonstrate statistically significant linkage.

\section{Microbiology}

Twenty-five separate microbiological samples were processed through the NSW Health Pathology Wollongong Hospital laboratory for the 26 cases. Two cases had isolates through external pathology providers that were not available for analysis. Twelve of the 25 samples (48\%) were from sterile sites (blood, tissue, pleural fluid or deep operative aspirate) while $4 / 25$ samples (16\%) were sputum and 9/25 (36\%) were superficial swabs or pus. Direct microscopy with Gram stain, modified acid-fast and standard acid-fast stains was performed on

Figure 2: Relative proportions of clinical nocardiosis presentations with relative causative species for each class

0.40

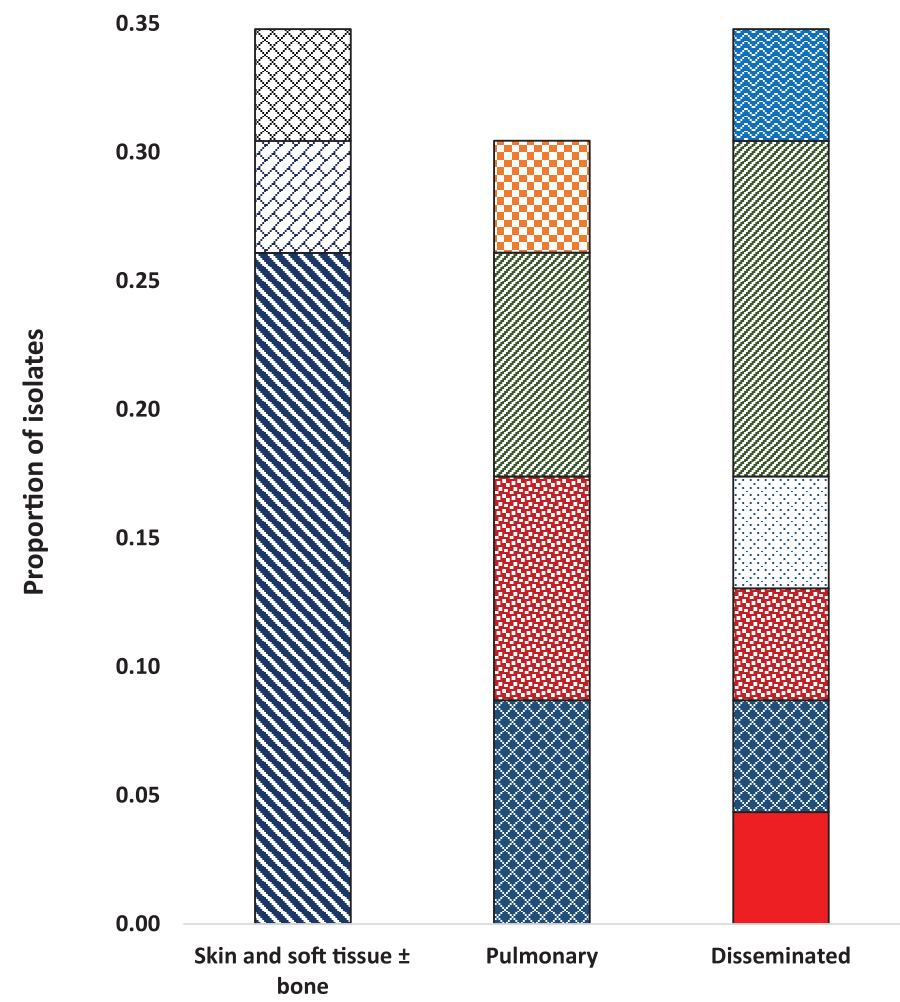

Nocardia sp. (gamkensis or exalbida)

$\otimes$ Nocardia thailandica

Nocardia rhamnosiphila

Nocardia beijingensis

Wocardia farcinica

Nocardia vinacea

图 Nocardia nova

Nocardia cyriacigeorgica

Nocardia paucivorans

$\mathbf{N}$ Nocardia brasiliensis 
Figure 3: Incidence of nocardiosis over the ten-year study period by postcode location ${ }^{\mathrm{a}, \mathrm{b}, \mathrm{c}}$

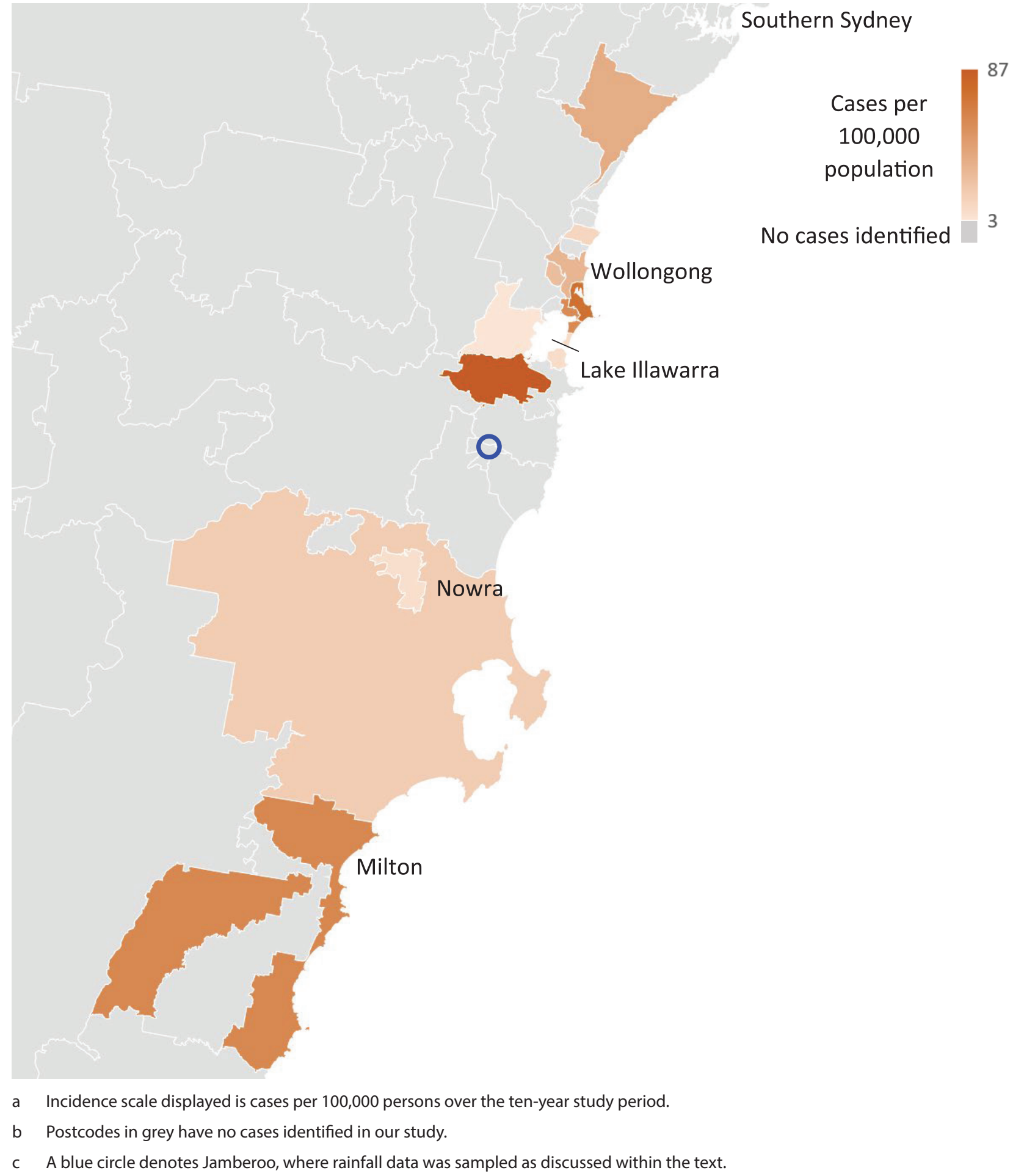

23/25 samples on either the day of sample collection or the following day. In 10/23 samples (43\%), organisms consistent with Nocardia species were seen on this examination.

Twenty-two of the 25 isolates (88\%) grew on conventional bacteriological media as described in the methods above. Eleven of the 25 culture-positive samples (44\%) were inoculated onto mycobacterial culture media (Lowenstein Jensen media) of which 3/11 (37\%) grew the Nocardia isolate. In all three of these samples Nocardia grew solely on mycobacterial culture media. The median time from collection to growth detection for all isolates was three days; however, this was skewed by isolates grown 
Figure 4: Incidence of Nocardia sp. infection in the Illawarra-Shoalhaven region (histogram) compared to total annual rainfall in Jamberoo (dashed line) ${ }^{\mathrm{a}}$

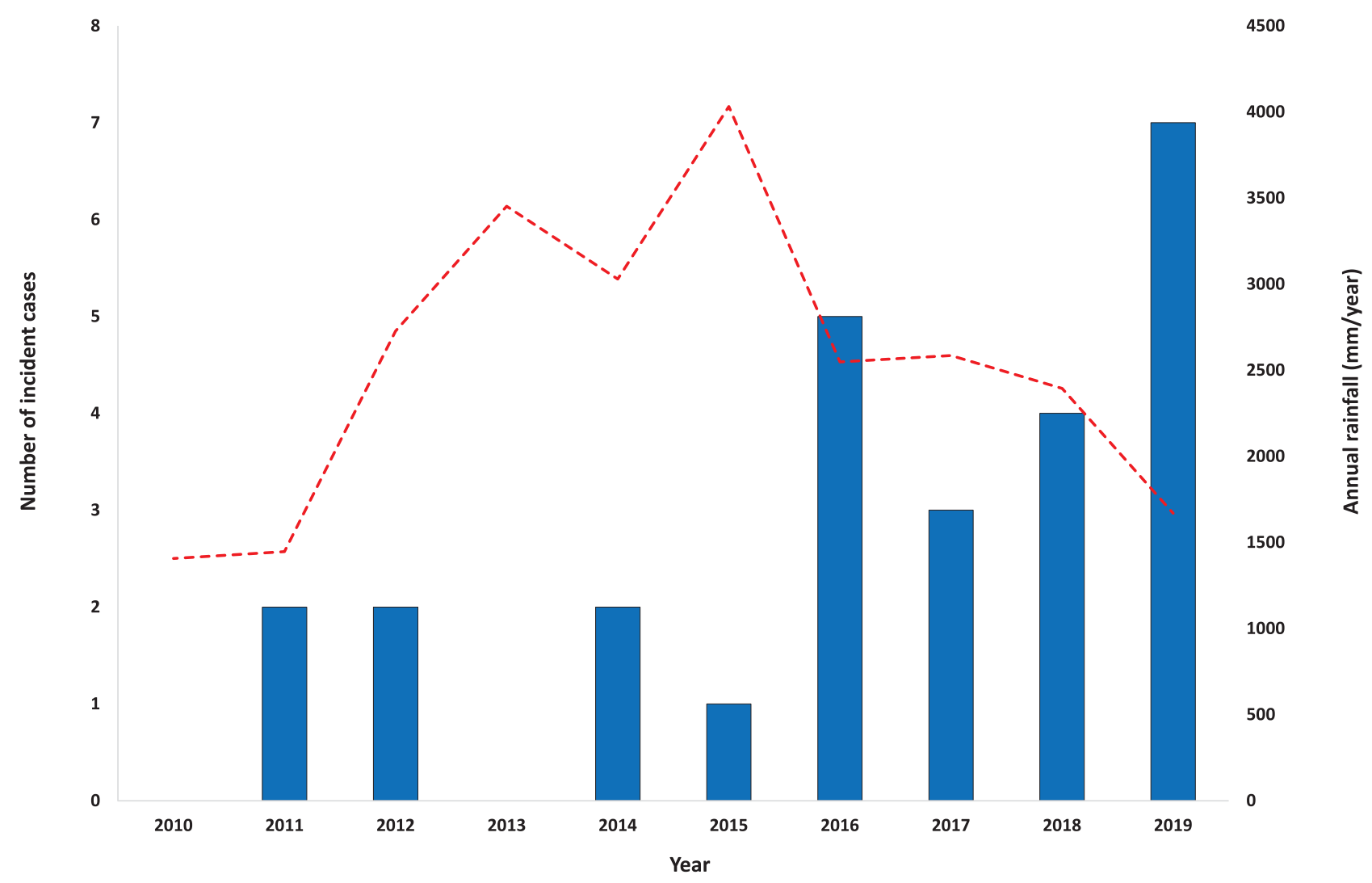

a These trends have a Pearson product-moment correlation coefficient of -0.34 (did not reach statistical significance; $p=0.2$ ).

only on mycobacterial culture media, which grew after a median of 34 days as opposed to those isolates cultured on routine bacteriological media which had a median time to growth detection of two days. Nocardia species were isolated from blood cultures in two cases.

Species identification was determined using 16S rRNA sequencing in 10/25 samples (40\%), MALDI-TOF MS in 10/25 (40\%) and using both methods in 5/25 (20\%).

\section{Antibiograms}

Antimicrobial susceptibility testing of isolates showed all were susceptible to trimethoprimsulfamethoxazole, amikacin and, where examined ( $n=11)$, linezolid; however, a high proportion were resistant to ciprofloxacin and carbapenems (Figure 5). Analysis of resistance patterns for species with more than one isolate (Table 3) demonstrates significant variability in resistance patterns between isolates (especially to ciprofloxacin and amoxicillin-clavulanate) even within the former $N$. asteroides complex.

\section{Antimicrobial therapy}

Data relating to treatment were available for 25 of the 26 cases identified. Initial therapy was with a single antimicrobial agent in $28 \%$ (7/25), two antimicrobials in 52\% (13/25), and three antimicrobials in $20 \%(5 / 25)$ of cases. Twenty-four of 25 patients (96\%) were treated with trimethoprim-sulfamethoxazole following identification of Nocardia sp. in diagnostic samples. The most commonly used agents, in addition to trimethoprim-sulfamethoxazole as part of an initial empiric combination therapy, were a carbapenem $(61 \% ; 11 / 18)$, ceftriaxone $(27 \% ; 5 / 18)$ and ciprofloxacin $(16 \% ; 3 / 18)$. This initial combination therapy was given for a median duration of ten days in skin and soft tissue disease, 28 days in pulmonary disease and 
Figure 5: In vitro antibiogram for all Nocardia isolates included in the study with antimicrobial susceptibility available $(\mathrm{N}=25)^{\mathrm{a}}$

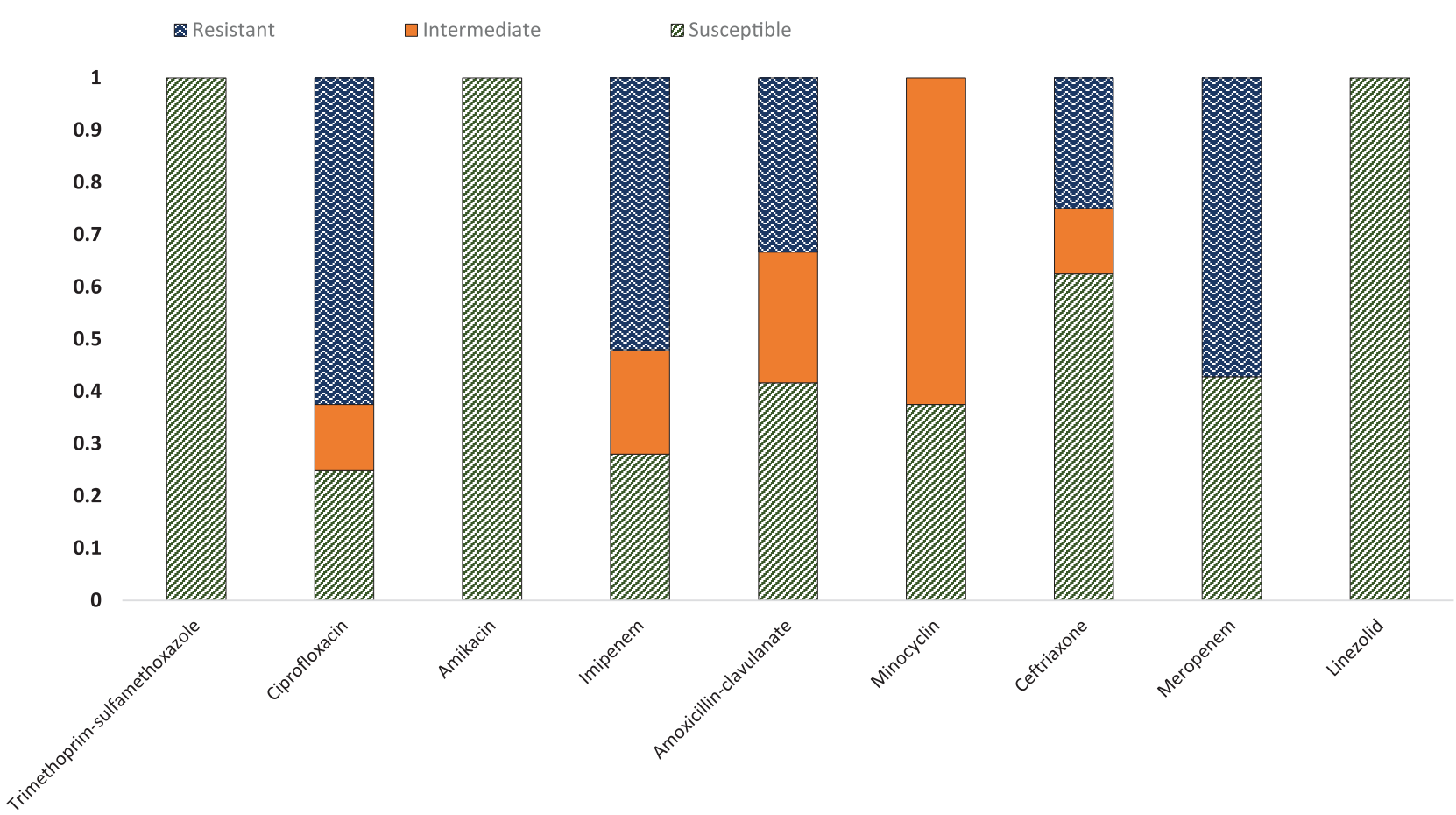

a All isolates had antibiotic susceptibility testing for trimethoprim-sulfamethoxazole, ciprofloxacin, amikacin, imipenem, amoxicillinclavulanate, minocycline and ceftriaxone. Only 7 isolates had susceptibility testing for meropenem reported and 11 isolates had susceptibility testing for linezolid performed.

six weeks in the case of disseminated or central nervous system disease. Monotherapy was most commonly trimethoprim-sulfamethoxazole $(85 \% ; 6 / 7)$.

Consolidation therapy (defined as a long-term maintenance or eradication treatment to prevent relapse) was with a single drug in $64 \%$ of cases (16/25) and with dual therapy in 36\% $(9 / 25)$. The single agent used most commonly was trimethoprim-sulfamethoxazole (88\%; 14/16). All dual-therapy regimens contained trimethoprim-sulfamethoxazole with, most commonly, the addition of either a tetracycline (doxycycline or minocycline) $(56 \%$; 5/9) or moxifloxacin $(33 \% ; 3 / 9)$. This consolidation therapy was given for a median duration of three months in skin and soft tissue disease, six months in pulmonary disease, and 12 months in disseminated or CNS disease.
Overall, $44 \%(11 / 25)$ of the patients with data available had documented evidence of an adverse drug reaction. This comprised acute kidney injury in $16 \%$ of cases $(4 / 25)$; gastrointestinal disturbance in $12 \%(3 / 25)$; liver function derangement in $8 \%(2 / 25)$; and cytopenia in $8 \%(2 / 25)$.

\section{Mortality statistics}

One-month mortality was $3.8 \%(1 / 26)$, whereas six-month mortality and 12-month mortality were $9.1 \%(2 / 22)$ and $15.7 \%(3 / 19)$ respectively. Only one patient died during initial therapy. No deaths were observed in the cohort with skin and soft tissue disease; however, several of these patients were lost to follow up.

\section{Discussion}

This retrospective study reports the demographic, clinical and microbiological 


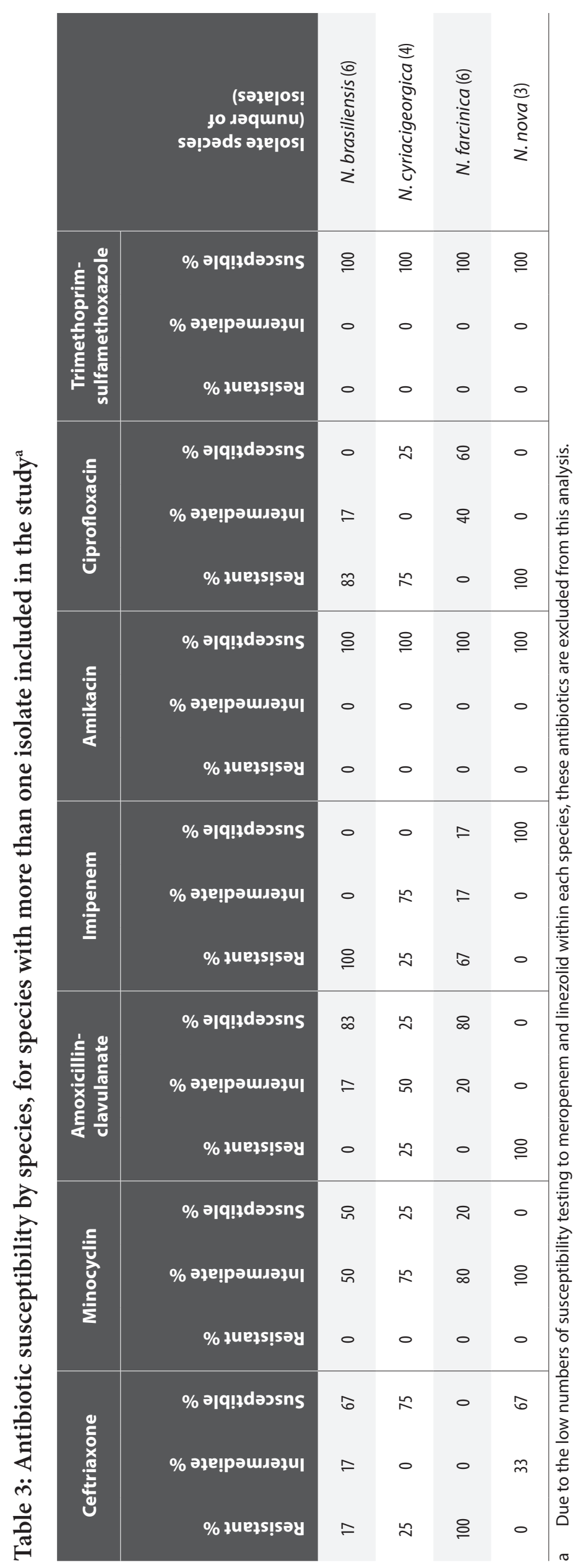


characteristics of 26 patients with cultureproven Nocardia infection over a ten-year period in a large and geographically diverse health service in regional NSW.

Comparison with other large Australian cohort studies demonstrates regional variation in the demographic characteristics and risk profile of patients with nocardiosis. Our cohort had similar demographic features to other studies of patients with nocardiosis in southern and eastern Australia, ${ }^{5}$ whereas nocardiosis patients in the Northern Territory were more likely to be younger, female, and of Aboriginal or Torres Strait Islander heritage. ${ }^{3}$

The majority of patients in this study (58\%) were immunocompromised, most commonly due to corticosteroid use (42\%), non-corticosteroid immunosuppressive medication (23\%) and/or haematological malignancy (19\%); solid organ transplantation was identified as a risk factor in just three patients in our cohort (12\%). By comparison, fewer than half of the patients in similar Australian nocardiosis studies were considered immunocompromised. High rates of chronic disease, including chronic lung disease, diabetes mellitus and end-stage renal disease, are commonly reported in nocardiosis studies; however, our study is notable for a much lower proportion of patients with chronic lung disease than other Australian nocardiosis studies (19\% vs $>50 \%)^{3,5}$

Given the variation in demographics and risk profile, it is unsurprising that the clinical characteristics of infections due to Nocardia sp. were also quite different. Paige and Spelman reported on 67 patients with nocardiosis, of whom 53 (79\%) had isolated pulmonary disease and just eight (12\%) had disseminated disease. ${ }^{5}$ In contrast, eight patients in our study (31\%) had isolated pulmonary disease and nine (35\%) had disseminated disease. This likely reflects the above-mentioned lower proportion of patients with chronic lung disease in our study and the higher proportion of patients with immunocompromise. Furthermore, in approximately one-third of patients in our study, nocardiosis manifested as isolated skin and soft tissue infection, often with a history of trauma at the site that may have resulted in inoculation of environmental organisms; all of which had a clinical syndrome consistent with Nocardia sp. infection. In contrast, only $7 \%$ of patients in the Victorian study had Nocardia infection limited to soft tissues and bone.

Antimicrobial susceptibility testing results in our study showed universal susceptibility of Nocardia sp. to trimethoprim-sulfamethoxazole and, where examined, linezolid, in keeping with other laboratory studies from NSW. ${ }^{12}$ This finding provides additional support for current Australian guidelines for the antimicrobial treatment of Nocardia sp. infection. ${ }^{13}$ It is also consistent with our treatment data showing that trimethoprim-sulfamethoxazole formed the backbone of most therapeutic regimens.

It should be noted that as ISLHD serves a large geographical area with a wide range of terrain and population density and that the majority of cases occurred in patients living away from the more densely populated centres in the northern Illawarra.

The majority of cases of nocardiosis reported here $(18 / 26)$ were identified in the last four years of the ten-year study, coinciding with a significant drought event in south-eastern Australia. ${ }^{14}$ Other Australian studies have hypothesised that the incidence of nocardiosis may be influenced by climatic conditions. ${ }^{7}$ In addition, international evidence suggests an association with dry, warm climates, for example in the south-eastern United States of America. ${ }^{1}$ Our study was unable to demonstrate a statistically significant correlation between the incidence of nocardiosis in ISLHD and regional rainfall trends; however, the study was likely underpowered for this purpose. In addition, for a large geographic area, complete rainfall data was only available for a single, albeit central, area of the region. This limited the resolution of analysis. 
This study was limited in its ability to sufficiently resolve trends in nocardiosis epidemiology by the small sample size. Furthermore, the limitations of retrospective data collection from clinical records (particularly with regards to treatment data) prevented more extensive analysis of the rationale and timing of changes to the treatment regimens for this uncommon disease.

We hypothesise that the increasing prevalence of patients with chronic comorbidities and immunocompromise, living in rural and regional communities exposed to environmental sources of Nocardia sp., may lead to an increased incidence of nocardiosis in the Illawarra-Shoalhaven region and that comparable trends may be seen in other regions with similar demographics and epidemiology. Larger prospective studies are required to test this hypothesis. The association between climatic conditions and incidence of nocardiosis remains unproven, but given the potential implications of global climate change, this concept warrants further investigation.

\section{Author details}

Dr Darcy Gray ${ }^{1}$

Dr Simeon Crawford ${ }^{1}$

Dr Peter Newton ${ }^{2}$

1. Department of Infectious diseases, Wollongong Hospital, Wollongong NSW 2500.

2. Microbiology, NSW Health Pathology, Wollongong Hospital, Wollongong NSW, 2500.

\section{Corresponding author}

Dr Darcy Gray

Address: Wollongong Hospital, Loftus Street, Wollongong, NSW 2500

Email: darcyrobert.gray@health.nsw.gov.au Phone: +61 437991575 


\section{References}

1. Saubolle MA, Sussland D. Nocardiosis: review of clinical and laboratory experience. J Clin Microbiol. 2003;41(10):4497-501.

2. Wilson JW. Nocardiosis: updates and clinical overview. Mayo Clin Proc. 2012;87(4):403-7.

3. McGuinness SL, Whiting SE, Baird R, Currie BJ, Ralph AP, Anstey NM et al. Nocardiosis in the Tropical Northern Territory of Australia, 1997-2014. Open Forum Infect Dis. 2016;3(4). doi: https://doi.org/10.1093/ofid/ofw208.

4. Rafiei N, Peri AM, Righi E, Harris P, Paterson DL. Central nervous system nocardiosis in Queensland. Medicine (Baltimore). 2016;95(46). doi: https://doi.org/10.1097/MD.0000000000005255.

5. Paige EK, Spelman D. Nocardiosis: 7-year experience at an Australian tertiary hospital. Intern Med J. 2019;49(3):373-9.

6. Roberts SA, Franklin JC, Mijch A, Spelman D. Nocardia infection in heart-lung transplant recipients at Alfred Hospital, Melbourne, Australia, 1989-1998. Clin Infect Dis. 2000;31(4):968-72.

7. Kennedy KJ, Chung KHC, Bowden FJ, Mews PJ, Pik JHT, Fuller JW et al. A cluster of nocardial brain abscesses. Surg Neurol. 2007;68(1):43-9.

8. Australian Bureau of Statistics. 2016 Census Quick Stats: Illawarra. [Webpage.] Canberra: Australian Bureau of Statistics; 23 October 2017. [Accessed on 23 April 2019.] Available from: https:// quickstats.censusdata.abs.gov.au/census_services/getproduct/census/2016/quickstat/107.

9. Australian Bureau of Statistics. 2016 Census Quick Stats: Southern Highlands and Shoalhaven. [Webpage.] Canberra: Australian Bureau of Statistics; 23 October 2017. [Accessed on 23 April 2019.] Available from: https://quickstats.censusdata.abs.gov.au/census_services/getproduct/census/2016/quickstat/114.

10. Australian Bureau of Statistics. 2016 Census Quick Stats: Australia. [Webpage.] Canberra: Australian Bureau of Statistics; 23 October 2017. [Accessed on 23 April 2019.] Available from: https:// quickstats.censusdata.abs.gov.au/census_services/getproduct/census/2016/quickstat/036.

11. Bureau of Meteorology. Monthly Rainfall. Jamberoo (The Ridge). All years of data. [Webpage.] Canberra: Australian Government Bureau of Meteorology. [Accessed on 3 May 2020.] Available from: http://www.bom.gov.au/jsp/ncc/cdio/wData/wdata?p_nccObsCode=139\&p_display_ type $=$ dataFile\&p_stn_num $=068035$.

12. Tan YE, Chen SCA, Halliday CL. Antimicrobial susceptibility profiles and species distribution of medically relevant Nocardia species: results from a large tertiary laboratory in Australia. J Glob Antimicrob Resist. 2020;20:110-7.

13. Therapeutic Guidelines. eTG complete: Nocardiosis. [Webpage.] Melbourne: Therapeutic Guidelines Limited; April 2019. Available from: https://tgldcdp.tg.org.au/viewTopic?topicfile=nocardios is. 
14. Bureau of Meteorology. Special Climate Statement 70 update-drought conditions in Australia and impact on water resources in the Murray-Darling Basin. Canberra: Australian Government Bureau of Meteorology; 13 August 2020. Available from: http://www.bom.gov.au/climate/current/statements/scs70.pdf. 